\title{
PROJETO EXPERIMENTAL DE UMA PLACA DE CONDICIONAMENTO DE SINAL PARA UM SENSOR DE CORRENTE DIFERENCIAL TIPO BOBINA DE ROGOWSKI ${ }^{1}$
}

\author{
Débora Debiaze de Paula² \\ Andreyna Sárila Ramos Ferreira \\ Paulo Jefferson Dias de Oliveira Evald \\ Cláudio Dornelles Mello Júnior \\ Rodrigo Zelir Azzolin
}

\begin{abstract}
RESUMO
Realizar a medição de grandezas físicas, tais como corrente e tensão, com precisão é de fundamental importância na área da instrumentação para automação, visto que tais medições geralmente realimentam controladores em malha fechada. De modo geral, os sinais aquisitados são de baixa intensidade. Assim, é indispensável o uso de placas de condicionamento de sinal para converter a grandeza medida em uma forma adequada aos sistemas de processamento de dados e controle. Dessa forma, a principal contribuição deste trabalho é apresentar uma metodologia para elaboração de um sistema de condicionamento de sinais para leitura de corrente, por meio de um sensor do tipo Bobina de Rogowski. Assim, espera-se que, como resultado deste trabalho, qualquer pessoa consiga reproduzir o sistema de medição bem como as placas de circuito impresso apresentadas neste documento para a correta medição de corrente. Além disso, também são apresentados, de forma didática, os passos matemáticos que atendam as especificações do projeto de instrumentação proposto.
\end{abstract}

Palavras-chave: Condicionamento de sinal. Bobina de Rogowski. Medição de corrente. Instrumentação.

\section{EXPERIMENTAL PROJECT OF A SIGNAL CONDITIONING PLATE FOR A ROGOWSKI COIL DIFFERENTIAL CURRENT SENSOR}

\begin{abstract}
Measurement of physical quantities, such as current and voltage, with precision is of fundamental importance in the area of instrumentation for automation, since such measurements generally feedback closed loop controllers. In general, the signals acquired have low intensity. Thus, it is essential to use signal conditioning plates to convert the measured quantity into a form suitable for data processing and control systems. Thus, the main contribution of this work is to present a methodology for the elaboration of a signal conditioning system for current reading, in a didactic way. It is expected that, as a result of this work, anyone can reproduce the measurement system, as well as the printed circuit boards presented in this document for correct current measurement. In addition, the mathematical

\footnotetext{
${ }^{1}$ Como citar este artigo: PAULA, D. D. de et al. Projeto experimental de uma placa de condicionamento de sinal para um sensor de corrente diferencial tipo Bobina de Rogowski. ForScience, Formiga, v. 8, n. 2, e00797, jul./dez. 2020. DOI: 10.29069/forscience.2020v8n2.e797.

${ }^{2}$ Autor para correspondência: Débora Debiaze de Paula, e-mail: deboradebiaze@yahoo.com.br
} 
steps that meet the specifications of the proposed instrumentation project are also presented in a didactic way.

Keywords: Signal conditioning. Rogowski coil. Current measurement. Instrumentation.

\section{INTRODUÇÃO}

Em sistemas de automação tanto no âmbito industrial, quanto comercial e até mesmo domésticos, é fundamental dispor de um sistema de aquisição de dados com sensores confiáveis e de custo compatível com a aplicação (THOMAZINI; ALBUQUERQUE, 2005). Alguns sensores frequentemente empregados na mensuração de corrente elétrica são do tipo: Transformador de Corrente (TC), Resistor Shunt, Sensor de Efeito Hall e Bobina de Rogowski (KOON, 2002). Em especial, o sensor do tipo Bobina de Rogowski, tratado neste trabalho, possui algumas vantagens, dentre as quais se destaca a sua grande faixa de leitura, em relação ao resistor shunt, a qual vai desde mA até alguns kA (HIGASHI, 2006). A bobina não possui histerese, visto que o seu núcleo é composto de material não magnético. Além disso, esse tipo de sensor apresenta boa linearidade, não possui contato físico com o circuito e a indutância mútua não é dependente da corrente nem da frequência do sinal a medir (HIGASHI, 2006). Tal sensor foi utilizado em trabalhos de Li et al. (2008) e Farjah (2016).

Segundo Rosário (2005), o sensor é um dispositivo sensível que altera a sua característica física interna por causa de um fenômeno físico externo, sendo assim um conversor de energia. Ele altera seu comportamento sob a ação de uma grandeza física, podendo fornecer direta ou indiretamente um sinal que mostra essa grandeza e convertendo uma quantidade física em um sinal elétrico. Já, segundo Thomazini e Albuquerque (2005), nem sempre um sensor possui as características elétricas para ser utilizado em uma próxima etapa de operação. É comum que o sinal de saída do sensor seja tratado antes de sua leitura efetiva. Esse tratamento, normalmente, é feito por um circuito de interface de condicionamento, para produção de um sinal que consiga ser lido pelo controlador estipulado para a aplicação (THOMAZINI; ALBUQUERQUE, 2005).

Ainda, segundo Kester (2005), é comum um sensor não possuir a capacidade de ser conectado diretamente ao próximo estágio de operação, ou seja, ao equipamento que realiza a análise e processamento de seu sinal. Isso ocorre, pois, o sinal pode ser de baixa intensidade, carecendo de amplificação. Além disso, o sinal pode estar corrompido com ruídos, que devem ser filtrados. Ainda, a relação do sensor pode ser não linear e necessitar de uma linearização prévia para integrar os dados ao próximo estágio. Por fim, o sinal pode ser medido como uma 
variação de tensão e ter que ser transformado para uma variação de corrente correspondente, entre outras situações (BOLTON, 2009). Todas essas transformações podem ser designadas ao condicionador de sinais.

Desta forma, a proposta deste trabalho é desenvolver um circuito de condicionamento de sinal para um sensor de corrente, que é composto pelos seguintes subcircuitos: Amplificador de Instrumentação, Amplificador Integrador e Amplificador Somador Inversor, e descrevê-los, de forma didática, para facilitar sua reprodução por outros interessados. Para testar o protótipo, será demonstrada a calibração experimental do sensor, cujos resultados são apresentados e discutidos. Vale a pena destacar que este trabalho é a síntese de um dos capítulos originalmente escritos no Trabalho de Conclusão de Curso (PAULA, 2018).

\section{SENSOR DE CORRENTE}

O sensor de corrente utilizado é uma Bobina de Rogowski, também conhecida como sensor $d i / d t$ (LERM, 2012; MAGISTRALLI, 2013), que é uma forma segura e confiável para medir corrente elétrica em circuitos CA. A Bobina de Rogowski é formada por um núcleo toroidal, não magnético, que é colocado em torno do condutor (HIGASHI, 2006). O campo magnético produzido pela corrente alternada no condutor induz uma tensão na bobina (HIGASHI, 2006). O sensor gera uma tensão proporcional à derivada no tempo da corrente que passa pelo condutor, necessitando de integração posterior para converter o sinal $d i / d t$ de volta para o formato de $i(t)$ para processamento adicional (KOON, 2002; LERM, 2012). Seu princípio de funcionamento está fundamentado na Lei de Ampère e na Lei da Indução de Faraday-Lenz (MAGISTRALLI, 2013). O Quadro 1 mostra uma comparação entre alguns sensores de corrente na qual é possível notar as vantagens que a Bobina de Rogowski possui.

\begin{tabular}{|l|c|c|c|c|}
\hline $\begin{array}{l}\text { Tecnologia do sensor de } \\
\text { corrente }\end{array}$ & $\begin{array}{c}\text { Resistor } \\
\text { Shunt }\end{array}$ & $\begin{array}{c}\text { Transformador } \\
\text { de Corrente }\end{array}$ & $\begin{array}{c}\text { Sensor de } \\
\text { efeito Hall }\end{array}$ & $\begin{array}{c}\text { Bobina de } \\
\text { Rogowski }\end{array}$ \\
\hline Custo & Muito baixo & Médio & Alto & Baixo \\
\hline $\begin{array}{l}\text { Linearidade na faixa de } \\
\text { medida }\end{array}$ & Muito bom & Fraco & Pobre & Muito bom \\
\hline $\begin{array}{l}\text { Capacidade de medida de } \\
\text { alta corrente }\end{array}$ & Muito pobre & Bom & Bom & Muito bom \\
\hline Consumo de potência & Alto & Baixo & Médio & Baixo \\
\hline $\begin{array}{l}\text { Problemas com saturação } \\
\text { em DC/alta corrente }\end{array}$ & Não & Sim & Sim & Não \\
\hline $\begin{array}{l}\text { Variação da saída com a } \\
\text { temperatura }\end{array}$ & Médio & Baixo & Alto & Muito baixo \\
\hline Problema de offset em DC & Sim & Não & Sim & Não \\
\hline $\begin{array}{l}\text { Problema de histerese e } \\
\text { saturação }\end{array}$ & Não & Sim & Sim & Não \\
\hline
\end{tabular}




\section{PROJETO DO SISTEMA DE CONDICIONAMENTO DE SINAIS}

O elemento utilizado para o condicionamento de sinais foi um amplificador operacional $(a m p-o p)$, nas suas mais diversas configurações, visto que ele é a base para muitos módulos condicionadores de sinal. Conforme Boylestad (1984), um amp-op é um amplificador diferencial de ganho muito alto, com impedância de entrada muito alta e impedância de saída baixa. O circuito básico é formado utilizando-se um amplificador de diferença com duas entradas (positiva e negativa) e pelo menos uma saída.

O sensor utilizado para medição de corrente, do tipo di/dt, é mostrado na Figura 1. Ele foi projetado para medição de corrente elétrica de até 20A e já estava disponível (custo zero).

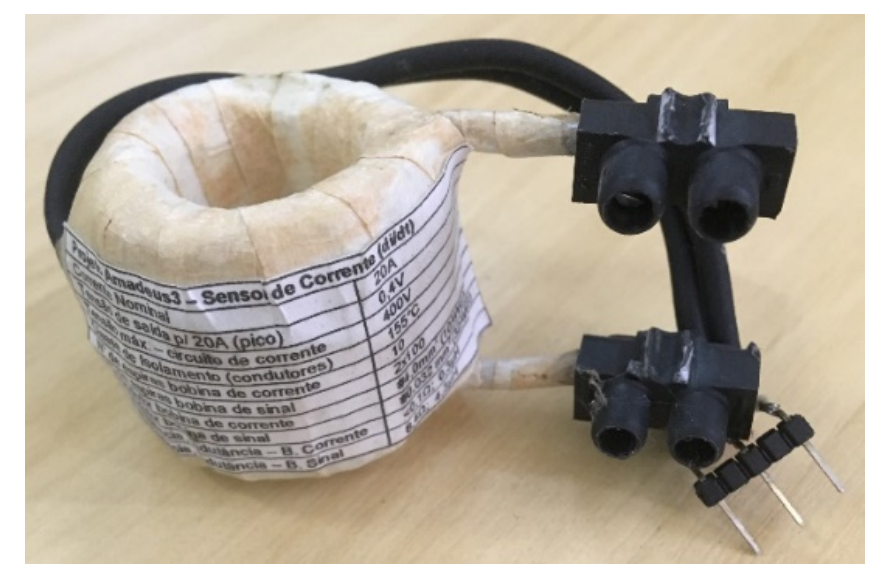

Figura 1 - Sensor de corrente $d i / d t$ utilizado Fonte: Autores (2018).

O circuito desenvolvido para o condicionamento de sinais, mostrado na Figura 2, é composto por três configurações de amplificadores operacionais, são eles: Amplificador de Instrumentação, Amplificador Integrador e Amplificador Somador Inversor. O circuito foi desenhado no software Eagle.

O primeiro estágio fornece uma saída em função da diferença entre seus dois sinais de entrada, sendo que o ajuste de ganho, que modifica a amplitude do sinal, é dado por Rganho_1. O segundo estágio funciona como um filtro passa-baixa, atenuando o ruído do sinal que vem do amplificador de instrumentação. Por fim, o terceiro estágio trata-se de um amplificador inversor somador, o qual combina o sinal filtrado com um nível CC, sendo ele o responsável por ajustar o offset do sinal, por meio do ganho Rganho_2. Vale a pena ressaltar que, os amp-ops são alimentados por uma fonte simétrica (vide Subseção 3.1). 


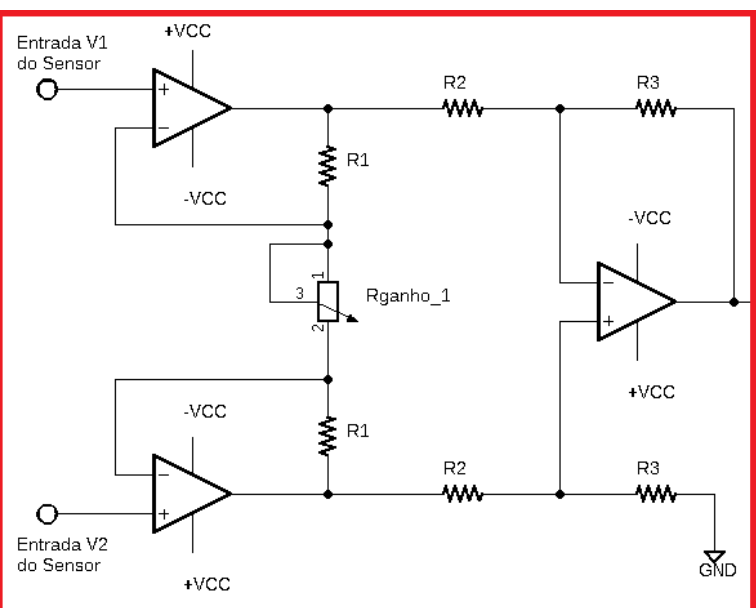

Amplificador de Instrumentação

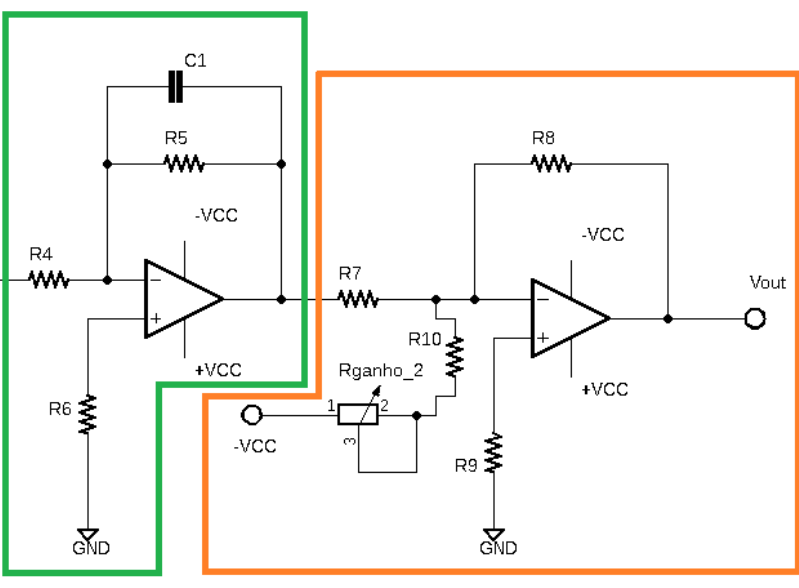

Amplificador Somador Inversor

Figura 2 - Circuito completo para o condicionamento de sinal Fonte: Autores (2018).

Conforme Pertence Jr. (2003), cada uma das três configurações apresentadas acima possui uma expressão que resulta em um ganho de tensão. Para o Amplificador de Instrumentação tem-se que:

$$
\begin{gathered}
G_{A I}=\frac{R_{3}}{R_{2}}\left(1+\frac{2 R_{1}}{\text { Rganho_1 }}\right) \\
G_{A I}=\frac{10 k}{10 k}\left(1+\frac{2 \times 1 k}{1 k}\right)=3
\end{gathered}
$$

Já o Amplificador Integrador:

$$
\begin{gathered}
G_{I}=\frac{R_{5} / R_{4}}{\sqrt{1+\left(2 \pi f R_{5} C_{1}\right)^{2}}} \\
G_{I}=\frac{150 k / 15 k}{\sqrt{1+(2 \pi 60 \times 150 k \times 10 n)^{2}}}=8,70
\end{gathered}
$$

Por fim, o Amplificador Somador Inversor é dado por:

$$
G_{S I}=-R_{8}\left(\frac{V_{\text {saída_integrador }}}{R_{7}}+\frac{\left(-V_{C C}\right)}{\left(R_{10}+\text { Rganho_2 }\right)}\right)
$$


O ganho do Amplificador Somador Inversor será proporcional a soma da tensão que sai do Amplificador Integrador, somada a referência de tensão $\left(-\boldsymbol{V}_{\boldsymbol{C C}}\right)$. Assim, a seguinte expressão é obtida:

$$
G_{S I}=-1 k\left(\frac{V_{\text {saída_integrador }}}{1,8 k}+\frac{\left(-V_{C C}\right)}{(4,7 k+\text { Rganho_2 })}\right)
$$

O ganho total $\left(G_{T}\right)$ é obtido por:

$$
G_{T}=G_{A I} \times G_{I} \times G_{S I}
$$

Na Tabela 1, é mostrada a descrição dos componentes utilizados na construção do circuito da Figura 2. De maneira geral, para se estipular os componentes do circuito da Figura 2, são necessárias especificações de projeto. Primeiramente é necessário saber qual a tensão máxima na entrada do equipamento que irá realizar a leitura da grandeza medida, sendo necessário também saber qual a corrente máxima que o equipamento medido fornece. Após isso, deve-se saber quanto de saída que a corrente máxima medida irá produzir no sensor, ou seja, a interface de medida de sinal não pode fornecer além do que a interface de leitura suporta. Desta forma, os ganhos são determinados de modo que a amplificação do sinal seja realizada. Basicamente, a maior parte dos resistores são atribuídos e o resistor de ajuste é calculado, de modo que o mesmo efetivamente realizará o ajuste do ganho.

\begin{tabular}{|c|c|c|c|}
\hline COMPONENTES & DESCRIÇÃO & COMPONENTES & DESCRIÇÃO \\
\hline$R_{1}$ & $1 \mathrm{k} \Omega$ & $R_{8}$ & $1 \mathrm{k} \Omega$ \\
\hline$R_{2}$ & $10 \mathrm{k} \Omega$ & $R_{9}$ & $1 \mathrm{k} \Omega$ \\
\hline$R_{3}$ & $10 \mathrm{k} \Omega$ & $R_{10}$ & $4,7 \mathrm{k} \Omega$ \\
\hline$R_{4}$ & $15 \mathrm{k} \Omega$ & $R_{\text {ganho_1 }}$ & $1 \mathrm{k} \Omega(102)$ \\
\hline$R_{5}$ & $150 \mathrm{k} \Omega$ & $R_{\text {ganho_2 }}$ & $5 \mathrm{k} \Omega(502)$ \\
\hline$R_{6}$ & $12 \mathrm{k} \Omega$ & $C_{1}$ & $10 \mathrm{nF}(103)$ \\
\hline$R_{7}$ & $1,8 \mathrm{k} \Omega$ & $A m p-O p$ & UA741 \\
\hline
\end{tabular}

Tabela 1- Descrição dos componentes utilizados no circuito

Fonte: Autores (2018).

O layout da placa para efetiva confecção de uma placa de circuito impresso é apresentado na Figura 3. 


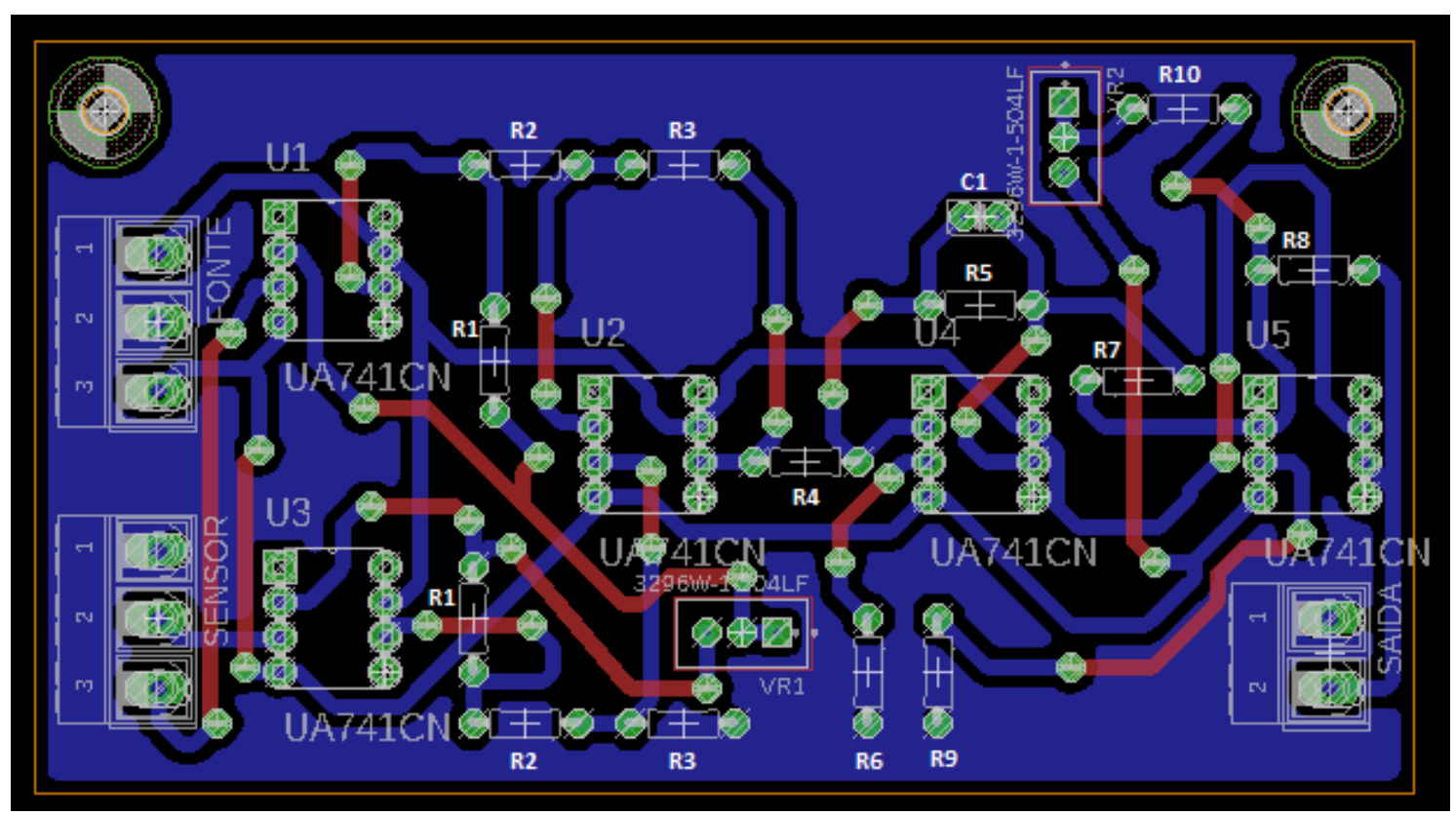

Figura 3 - Layout do circuito de condicionamento para o sensor de corrente Fonte: Autores (2018).

\subsection{Fonte simétrica}

Uma fonte de alimentação é um circuito encarregado por gerar uma tensão constante para um circuito eletrônico e fornece alimentação contínua a partir de uma alimentação alternada. Quando construída apropriadamente, uma fonte de alimentação deve garantir uma tensão contínua e estabilizada em sua saída, isto é, mesmo que a tensão de entrada sofra uma variação, a tensão de saída deverá se manter constante.

O transformador obtém $110 \mathrm{~V}$ (ou $220 \mathrm{~V}$ dependendo da ligação do transformador Figura 4) da rede elétrica no primário e rebaixa essa tensão no secundário para $\pm 15 \mathrm{~V}$, estas tensões são retificadas por uma ponte retificadora, conforme mostrado na Figura 5. Essa retificação é realizada para que tensões simétricas sejam atingidas em relação à saída do transformador, ou seja, um sinal de corrente alternada é modificado em corrente contínua impedindo que haja mudança de sentido do fluxo da corrente elétrica. 


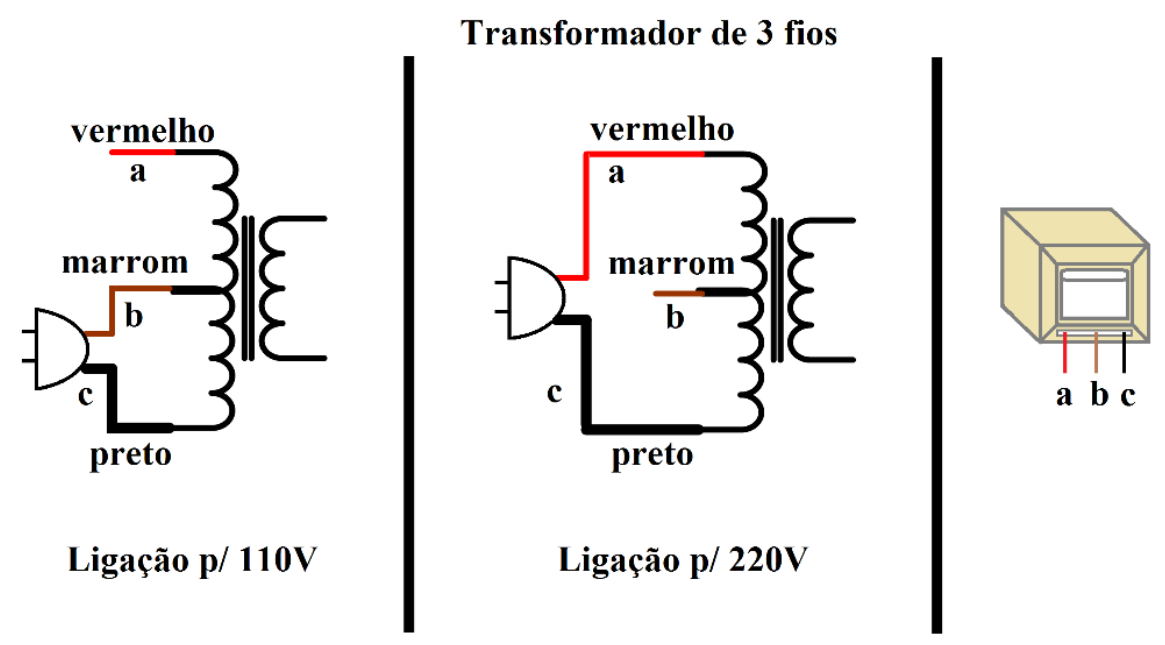

Figura 4- Ligação de um transformador de 3 fios Fonte: Adaptado de Eletrônica Phoenix (2018).

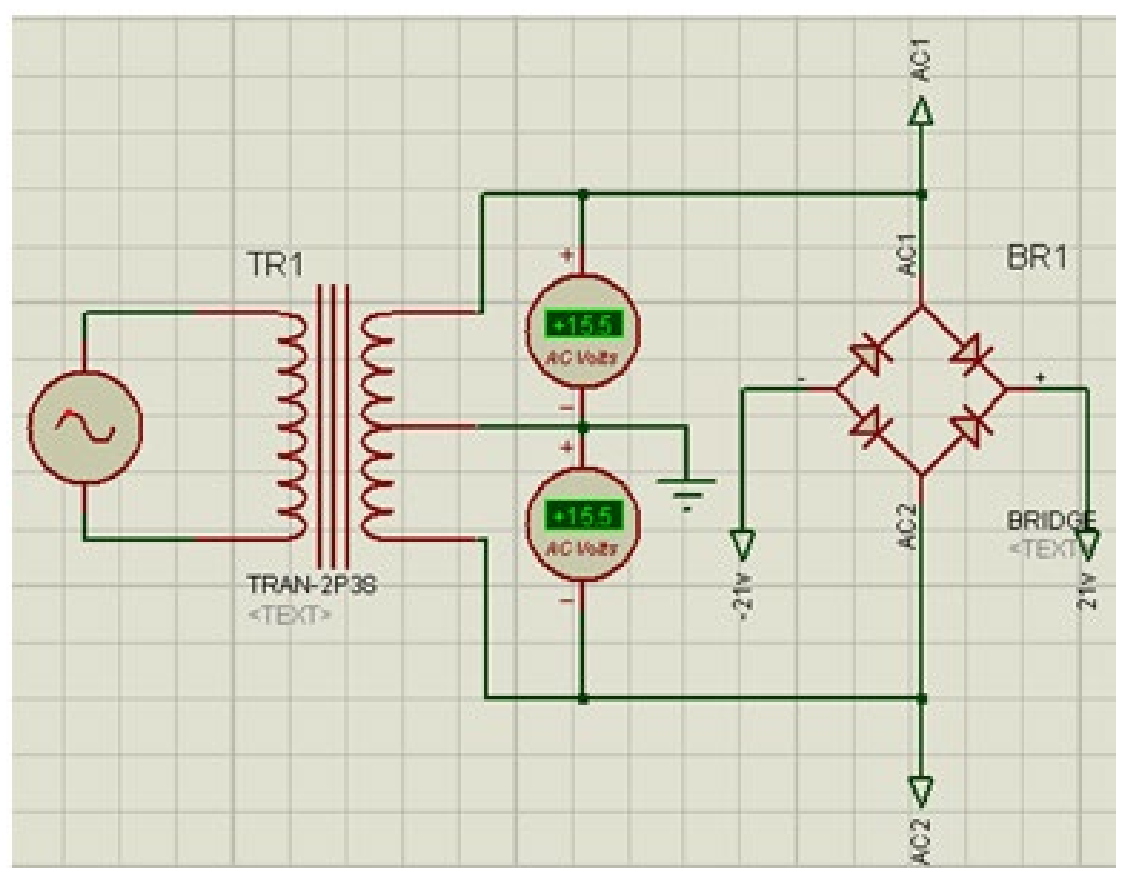

Figura 5 - Diagrama do transformador com ponte retificadora Fonte: Autores (2018).

A filtragem da tensão retificada é realizada por dois capacitores, um eletrolítico de $2200 \mu \mathrm{F} / 25 \mathrm{~V}$ e outro cerâmico de $100 \mathrm{nF}$. Já a regulagem das tensões é feita por circuitos integrados chamados de reguladores de tensão. A série 78 e 79 são os mais usuais destes tipos. O circuito integrado (CI) da linha 78 é aplicado em saídas positivas, conforme ilustrado na Figura 6 e o CI da linha 79 são aplicados em saídas negativas, conforme ilustrado na Figura 7. Na saída dos reguladores, existe um capacitor eletrolítico de $470 \mu \mathrm{F} / 25 \mathrm{~V}$ que serve para desviar possíveis pulsos de comutação rápidos, ou seja, ruídos. 


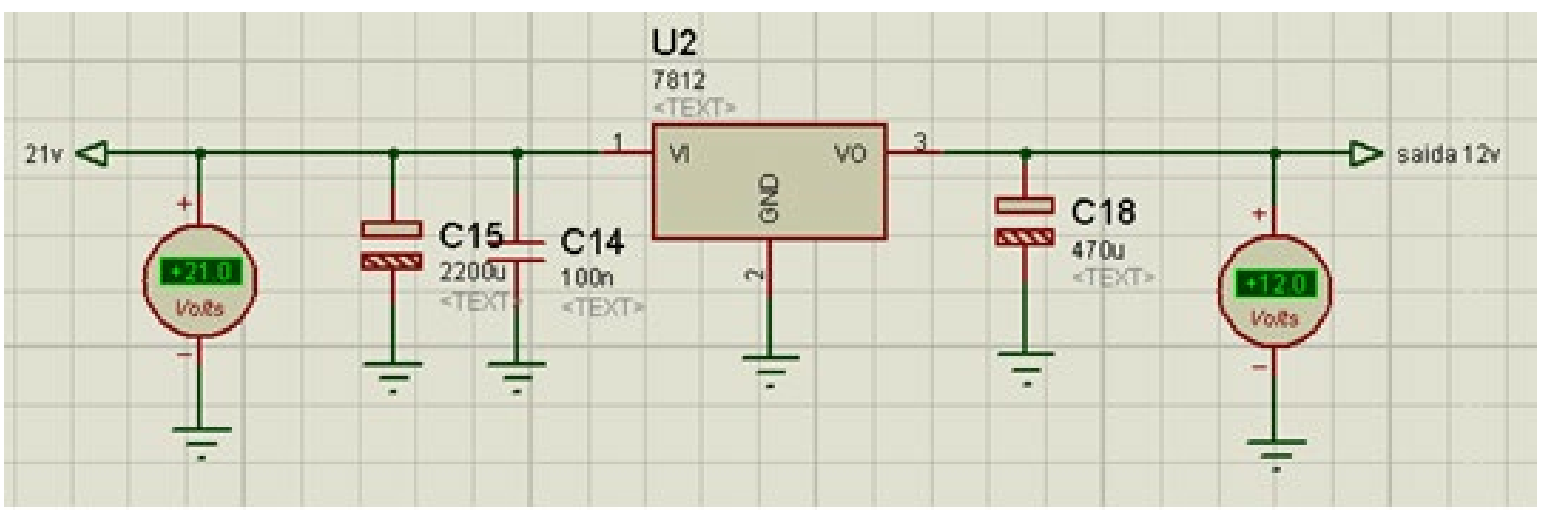

Figura 6 - Diagrama do regulador de tensão 7812 Fonte: Autores (2018).

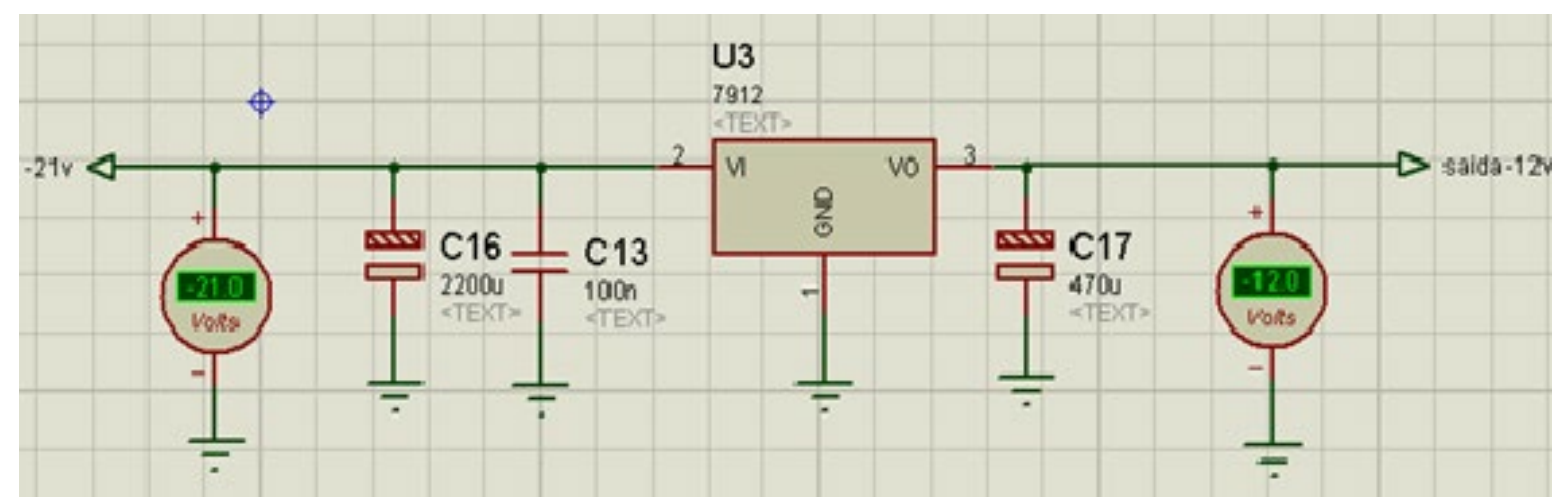

Figura 7- Diagrama do regulador de tensão 7912 Fonte: Autores (2018).

Foram utilizados os seguintes materiais para construção da fonte simétrica:

- 1x Transformador de $-15+15 \mathrm{~V} / 500 \mathrm{~mA}$;

- 1x Ponte Retificadora modelo MIC RS507 (DC, 2018);

- 1x CI 7812 (NATIONAL (TPR), 2018);

- 1x CI 7912 (NATIONAL (TNR), 1994);

- 2x Capacitores eletrolíticos de $2200 \mu \mathrm{F} / 25 \mathrm{~V}$;

- 2x Capacitores eletrolíticos de $470 \mu \mathrm{F} / 25 \mathrm{~V}$;

- 2x Capacitores cerâmicos de 100nF; e

- $2 \mathrm{x}$ Bornes.

Para construção apropriada da fonte simétrica de alimentação, foi desenvolvido o layout da placa, mostrado na Figura 8. 
PAULA, D. D. de et al. Projeto experimental de uma placa de condicionamento de sinal para um sensor de 10 corrente diferencial tipo Bobina de Rogowski

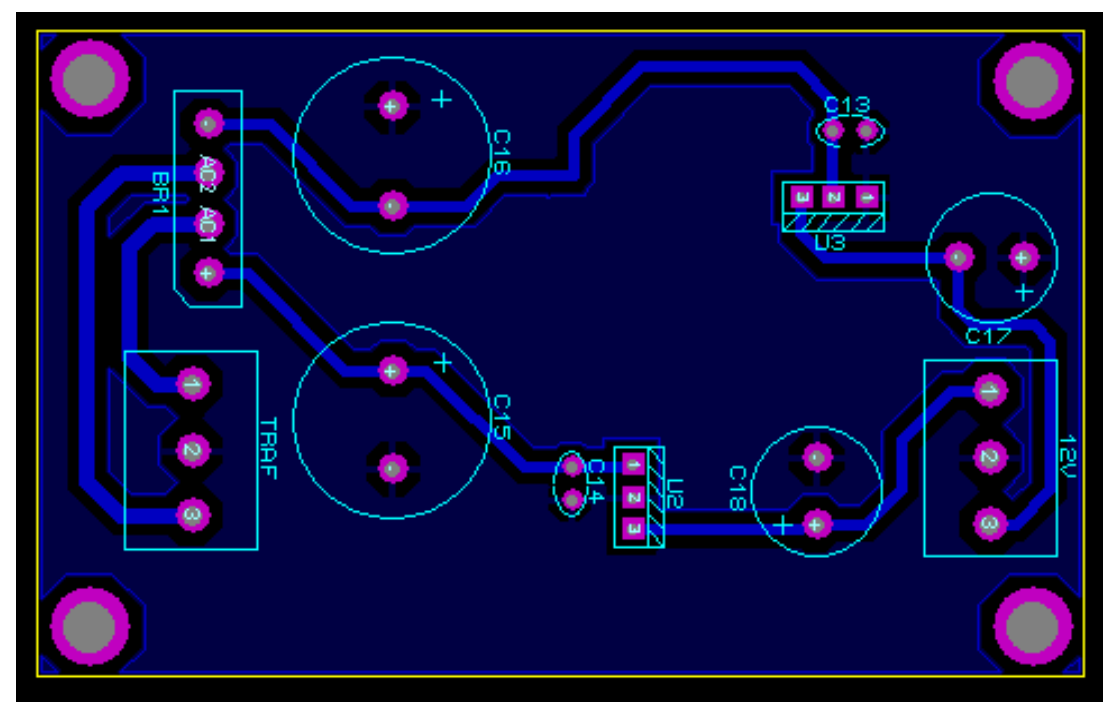

Figura 8 - Layout do circuito da fonte simétrica Fonte: Autores (2018).

Portanto, as características da fonte construída são:

- Tensão de entrada: 110V CA; e

- Tensão de saída: \pm 12 V CC.

\section{RESULTADOS EXPERIMENTAIS}

Após a elaboração do esquemático e layout da placa de condicionamento para o sensor de corrente, a placa foi confeccionada. Na Figura 9, é mostrado o protótipo construído.

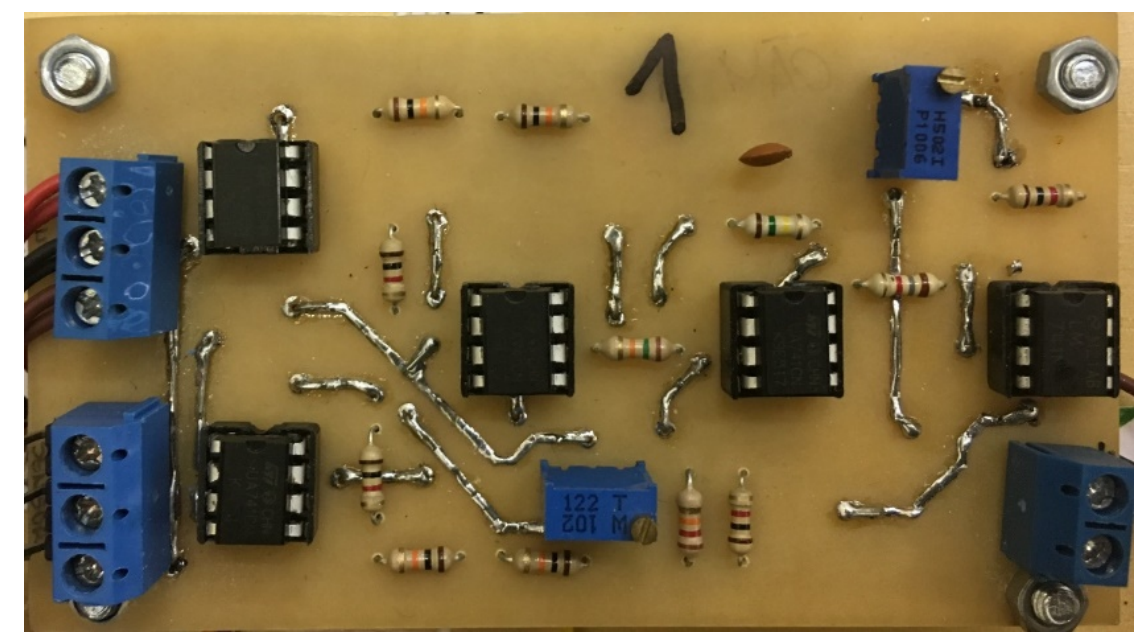

Figura 9 - Placa de condicionamento de sinal Fonte: Autores (2018). 
Visto que a corrente que será medida pelo sensor diferencial (Figura 1), possui característica senoidal e que este sinal será analisado e processado por um Arduino DUE, alguns aspectos devem ser levados em consideração para os ajustes da placa. Sabe-se que os pinos do Arduino DUE trabalham com uma faixa de tensão de 0 a 3,3 V, assim a amplitude da onda medida deve se manter dentro desta faixa, possuindo um offset de $1,65 \mathrm{~V}$, isto é $3,3 \mathrm{~V}$ pico a pico. Para tal, em um primeiro momento, o nível de corrente medido, aproximadamente 7 A, foi obtido colocando o sensor em série com uma carga resistiva. Com a saída da placa conectada a um osciloscópio, mostrado na Figura 10, percebe-se que tanto a amplitude, como o offset foram alcançados. Como discutido anteriormente, o trimpot Rganho_l é responsável pelo ajuste da amplitude e o trimpot Rganho_2 pelo ajuste do offset.

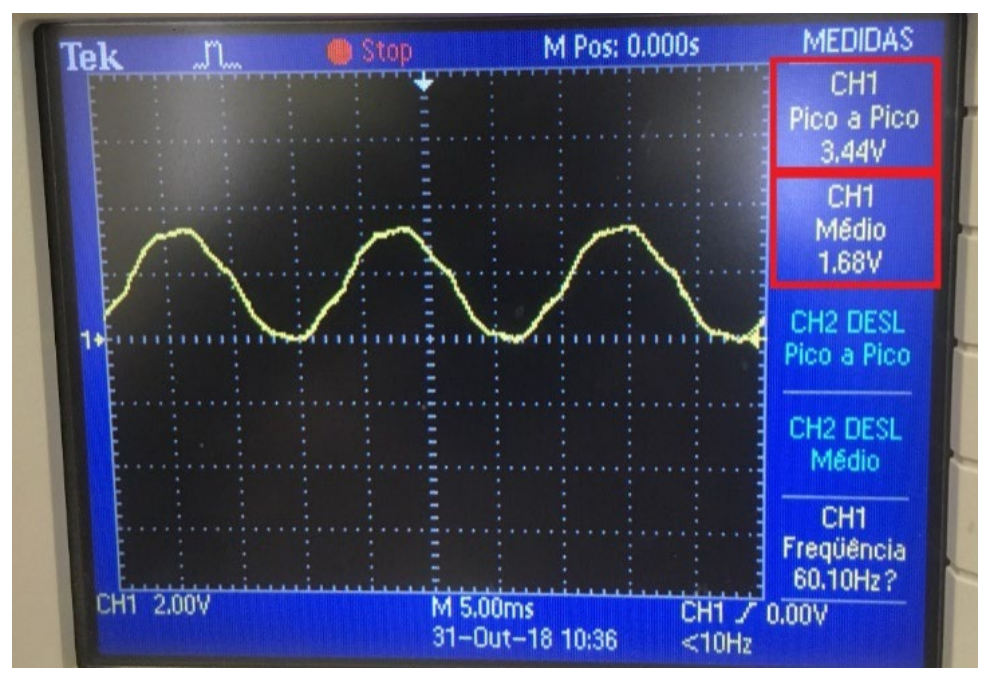

Figura 10- Ajuste de amplitude e offset do sinal Fonte: Autores (2018).

\subsection{Curva de calibração dos sensores de corrente}

Para o levantamento da curva de calibração dos sensores de corrente, um segundo experimento foi realizado com os seguintes equipamentos: Transformador Variável, Amperímetro, Voltímetro e Conjunto de Cargas Resistivas. Vale a pena destacar que serão medidas as correntes de duas fases do motor, pois foi suposto que o motor é equilibrado e que a corrente de neutro é zero. Se a corrente de neutro é zero, pela Lei de Kirchhoff das correntes, tem-se que o somatório das correntes das fases é igual a zero. Sendo assim, a seguinte relação é válida (BOYLESTAD, 1984):

$$
\begin{gathered}
I_{R_{\text {medido }}}+I_{S_{\text {medido }}}+I_{T}=0 \\
I_{T}=-I_{R_{\text {medido }}}-I_{S_{\text {medido }}}
\end{gathered}
$$


Portanto, foram construídas duas placas de condicionamento de sinais para o sensor de corrente. Contudo, os resultados discutidos a seguir são apenas para um conjunto sensor/placa, uma vez que o procedimento para ambas é idêntico. A disposição dos equipamentos para o experimento de levantamento da curva de calibração é mostrada na Figura 11.

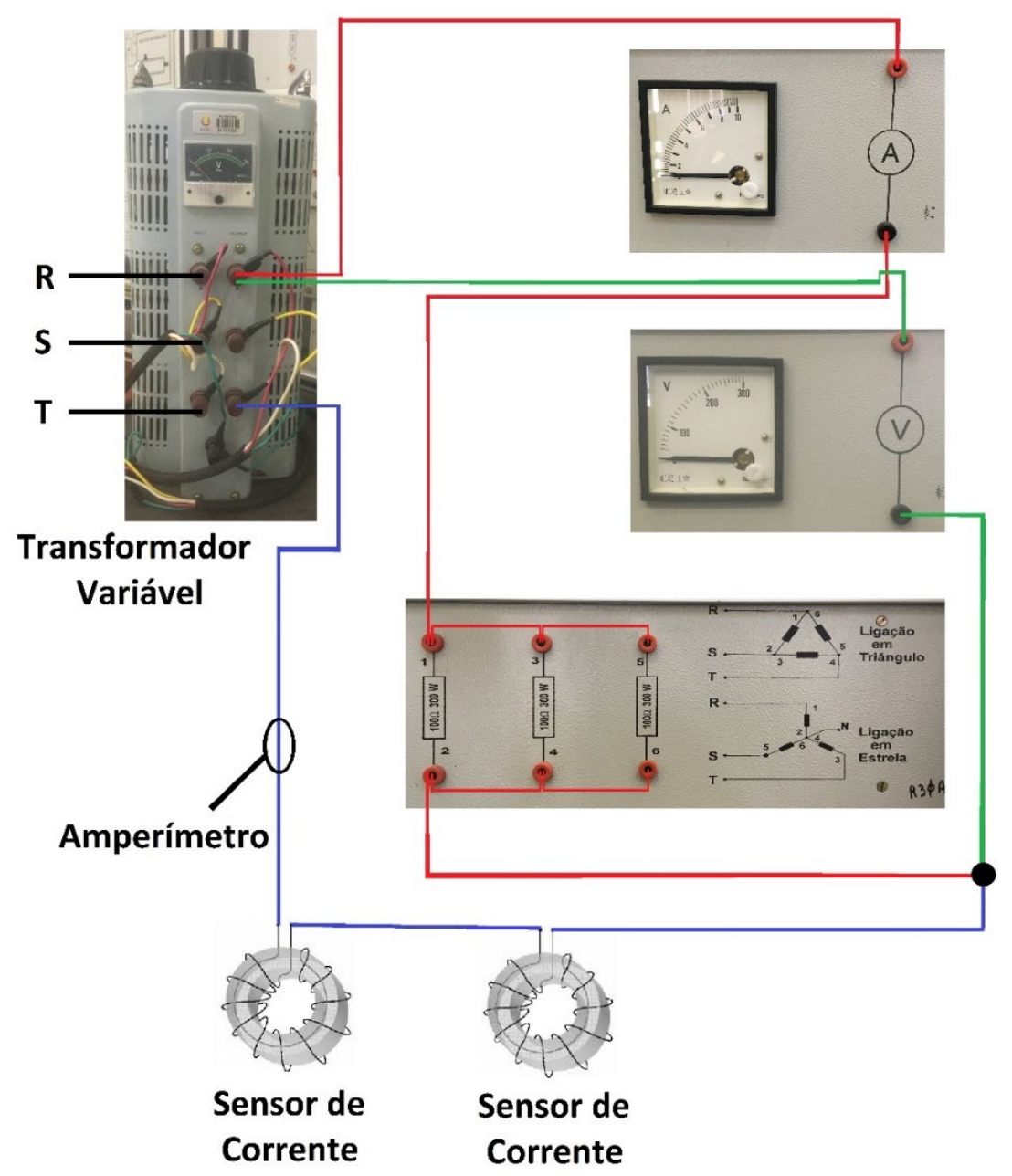

Figura 11- Disposição dos equipamentos para medição de corrente Fonte: Autores (2018).

Para obter a curva de calibração, é necessário realizar um conjunto de testes. Para tal, foram utilizados diferentes resistores e mensuradas as respectivas correntes, bem como os valores correspondentes lidos pelo Arduino. Na Figura 12, é mostrado esse conjunto de resistores, que são utilizados como carga. 
PAULA, D. D. de et al. Projeto experimental de uma placa de condicionamento de sinal para um sensor de

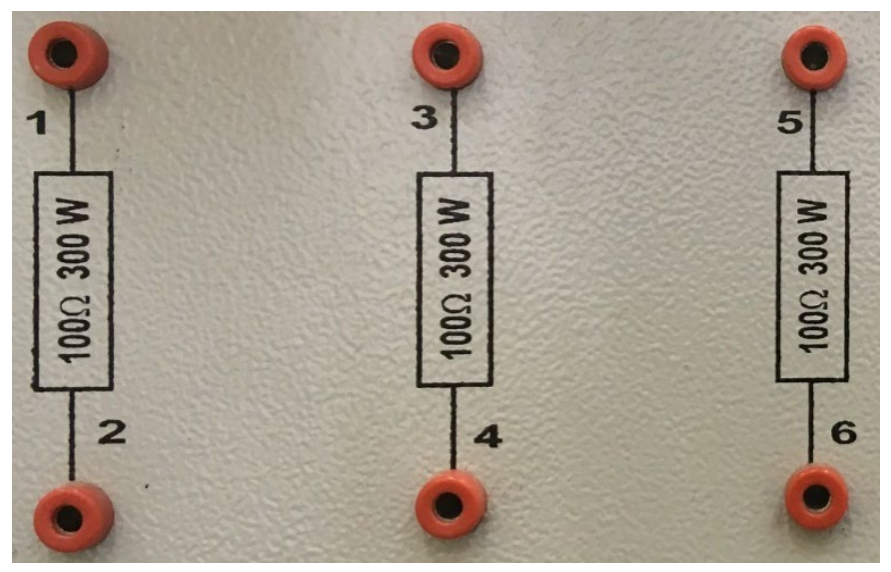

Figura 12 - Conjunto de resistores utilizados como carga Fonte: Autores (2018).

Cada resistor possui uma potência de $300 \mathrm{~W}$ e uma resistência de $100 \Omega$. Por meio da associação de resistores em paralelo, obtém-se uma resistência equivalente dada por (12).

$$
\begin{gathered}
\frac{1}{R_{e q}}=\frac{1}{R_{1}}+\frac{1}{R_{2}}+\frac{1}{R_{3}} \\
\frac{1}{R_{e q}}=\frac{3}{100} \\
R_{e q}=33,3 \Omega
\end{gathered}
$$

Sabendo-se que a potência total do circuito é $900 \mathrm{~W}$ e que a potência elétrica é dada por (13), a corrente elétrica é dada por (14).

$$
\begin{gathered}
P=R \times i^{2} \\
i=\sqrt{\frac{900}{33,3}}=5,2 A
\end{gathered}
$$

Desta forma, através da primeira Lei de Ohm é possível encontrar a tensão do circuito, dado por (15).

$$
V=R \times i=33,3 \times 5,2=173,16 V
$$


Assim, sabe-se que a corrente máxima que o sensor de corrente irá medir é de aproximadamente 5,2 A, sendo que só alcançará a tensão máxima de aproximadamente $173,16 \mathrm{~V}$, ao variar a tensão por meio do transformador variável. Para monitorar a corrente medida, foi conectado ao osciloscópio um amperímetro. Ele foi configurado para que cada $100 \mathrm{mV}$ correspondesse à $1 \mathrm{~A}$ medido.

Com toda bancada montada, um código de leitura foi desenvolvido para o controlador. Este não está incorporado ao artigo, por restrições de espaço. A Tabela 2 mostra os valores coletados.

Tabela 2 - Equivalência da corrente medida com a corrente lida pelo Arduino

\begin{tabular}{cc}
\hline $\begin{array}{c}\text { Corrente Medida } \\
(\text { I_med(A)) }\end{array}$ & $\begin{array}{c}\text { Leitura do Arduino } \\
(\text { I_arduino(A)) }\end{array}$ \\
\hline $\mathbf{0 , 5}$ & 0,3 \\
$\mathbf{1}$ & 0,63 \\
$\mathbf{1 , 5}$ & 0,98 \\
$\mathbf{2}$ & 1,28 \\
$\mathbf{2 , 5}$ & 1,6 \\
$\mathbf{3}$ & 2 \\
\hline
\end{tabular}

Fonte: Dos autores (2018).

Para levantar as curvas de calibração dos dois sensores, foi utilizado o algoritmo de interpolação linear. Desta forma, em (16) e (17) são mostradas as curvas encontradas para o conjunto sensor1/placal e o conjunto sensor $2 /$ placa2, respectivamente:

$$
\begin{aligned}
& y_{I_{\text {med }}}=1,5 \times x_{I_{\text {arduino }}}+0,061 \\
& y_{I_{\text {med }}}=1,4 \times x_{I_{\text {arduino }}}+0,075
\end{aligned}
$$

\subsection{Validação da curva de calibração dos sensores de corrente}

Esse procedimento foi realizado somente para verificar se a corrente medida pelo amperímetro era a mesma medida pelo Arduino, por meio da equação encontrada anteriormente. Na Figura 13 (a), é mostrada a corrente no osciloscópio e, em (b), os dados lidos pelo Arduino, por meio da ferramenta embarcada plotter. Note que os valores lidos pelo Arduino são equivalentes aos dados no osciloscópio, ou seja, a leitura de uma corrente de 2 A. 


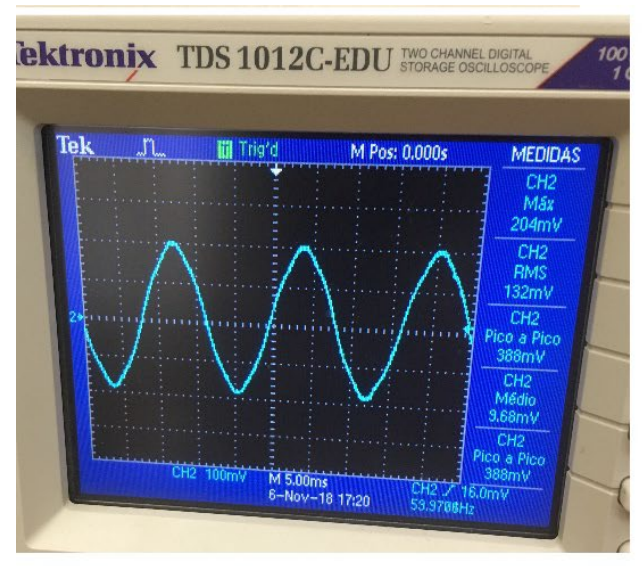

(a)

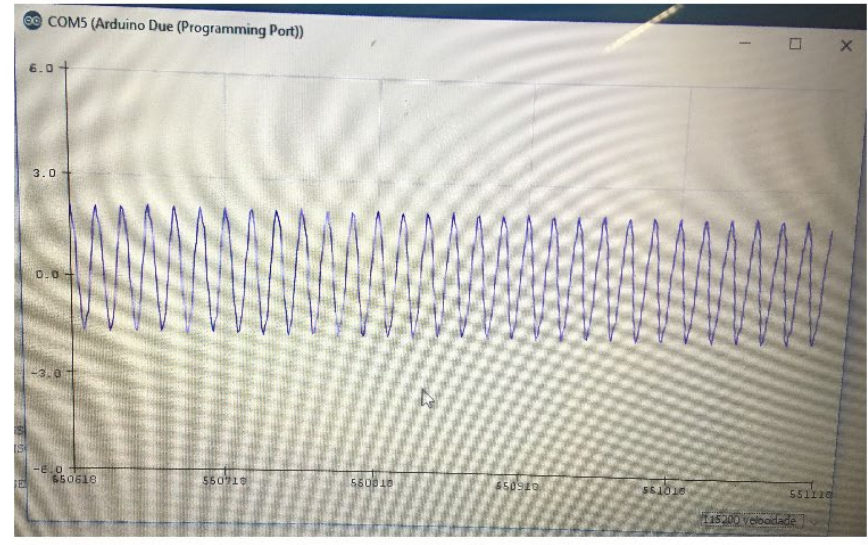

(b)

Figura 13 - Validação da curva de calibração, onde (a) Leitura do amperímetro e (b) Leitura do Arduino (ferramenta Plotter) Fonte: Autores (2018)

\section{CONCLUSÃO}

Neste trabalho, foi apresentada uma metodologia didática para desenvolver e construir uma interface de condicionamento de sinais para um sensor de corrente diferencial (di/dt), também conhecido como Bobina de Rogowski. Para permitir que o sinal seja processado pelo Arduino DUE, também foram mostrados os detalhes do projeto embarcado. Ainda, os aspectos construtivos e a validação experimental foram apresentados e discutidos. Além disso, foi mostrado que é possível realizar ajustes tanto na amplitude quanto no offset dos sinais por meio dos resistores variáveis.

\section{REFERÊNCIAS}

BOLTON, W. Mecatrônica: uma abordagem multidisciplinar. Porto Alegre: Bookman, 2009.

BOYLESTAD, R. L.; NASHELSKY, L. Dispositivos eletrônicos e teoria de circuitos. Prentice-Hall do Brasil, 1984.

DC COMPONENTS. Datasheet: RS507 Bridge Rectifier. Electronic Publication, 2018.

KESTER, W. Sensor signal conditioning. In: Sensor technology handbook. Newnes, 2005. p. 31-136.

ELETRÔNICA PHOENIX. Ligação transformador de 3 fios. Disponível em:

http://www.geocities.ws/eletronica_phoenix/transformadores1.htm. Acesso em: 25 jan. 2018.

FARJAH, E.; GIVI, H.; GHANBARI, T. Application of an efficient Rogowski coil sensor for switch fault diagnosis and capacitor ESR monitoring in nonisolated single-switch DC-DC converters. IEEE Transactions on Power Electronics, v. 32, n. 2, p. 1442-1456, 2016. 
HIGASHI, E. M. Modelagem da bobina de Rogowski para medidas de pulsos de corrente elétrica. 2006. 82 f. Mestrado (Mestrado em Engenharia) - Universidade Federal do Paraná, Curitiba, 2006.

KOON, W. Current sensing for energy metering. In: Conference Proceedings IICChina/ESC-China. 2002. p. 321-324.

LERM, R. Amadeus 3: arquitetura modular para a automatização da distribuição de energia. 2012. 51 f. Projeto de Graduação (Engenharia de Automação) - Universidade Federal do Rio Grande, Rio Grande, 2012.

LI, W. et al. Transient characteristics of Rogowski coil-based current sensor [J]. Electric Power Automation Equipment, v. 28, n. 10, p. 34-37, 2008.

MAGISTRALLI, G. Amadeus 3: supervisório para arquitetura modular para a automatização da distribuição de energia. 2013. 71 f. Projeto de Graduação (Engenharia de Automação) - Universidade Federal do Rio Grande, Rio Grande, 2013.

NATIONAL SEMICONDUTOR. Datasheet: terminal negative regulators (TNR). Electronic Publication, 1994.

NATIONAL SEMICONDUTOR. Datasheet: Terminal positive regulators (TPR). Electronic Publication, 2018.

PAULA, D. D. de. Projeto de sistema para acionamento e controle de motores de indução trifásicos. 2018. 162 f. Projeto de Graduação (Engenharia de Automação) - Universidade Federal do Rio Grande, Rio Grande, 2018.

PERTENCE JR, A. Amplificadores operacionais e filtros ativos. 6. ed. Porto Alegre: Bookman, 2003.

ROSÁRIO, J. M. Princípios de mecatrônica. Pearson Educación, 2005.

THOMAZINI, D.; ALBUQUERQUE, P. U. B. de. Sensores industriais: fundamentos e aplicações. São Paulo, v. 3, p. 32, 2005.

\section{DADOS DOS AUTORES:}

Nome: Débora Debiaze de Paula

E-mail: deboradebiaze@yahoo.com.br

Curriculum Lattes: http://lattes.cnpq.br/8195414525211156

Mestranda em Engenharia de Computação pela Universidade Federal de Rio Grande (FURG) e graduação em Engenharia de Automação pela mesma Instituição. Possui experiência nas áreas de Robótica, Controle de Sistemas, Automação e Eletrônica. Atualmente trabalha com robótica, processamento de imagens e aprendizado profundo em ambientes subaquáticos.

Nome: Andreyna Sárila Ramos Ferreira 
E-mail: andreynasrf@gmail.com

Curriculum Lattes: http://lattes.cnpq.br/0621391304599077

Mestranda em Engenharia Eletrônica e Computação no Instituto Tecnológico de Aeronáutica e graduação em Engenharia de Automação pela Universidade Federal do Rio Grande. Atua principalmente nas áreas de Eletrônica, Controle e Automação, envolvendo controle de sistemas e programação. Possui experiência em robótica industrial, aplicando técnicas de controle clássico e moderno com o objetivo de melhorar o comportamento de sistemas frente a variações de velocidade e posição. Atualmente trabalha com sistemas de controle na área aeronáutica.

Nome: Paulo Jefferson Dias de Oliveira Evald

E-mail: paulo.evald@gmail.com

Curriculum Lattes: http://lattes.cnpq.br/1946704909213416

Doutorando em Engenharia Elétrica na Universidade Federal de Santa Maria; mestrado em Engenharia de Computação e graduação em Engenharia de Automação, ambas pela Universidade Federal do Rio Grande (FURG). Atualmente, é Professor Assistente da Universidade Franciscana (UFN), lotado na Engenharia Biomédica. As principais áreas de interesse são divididas em 3 grupos: - Modelagem matemática de sistemas dinâmicos, controladores robustos adaptativos e controle aplicado a sistemas de energias renováveis; Engenharia de reabilitação e tecnologias assistivas; - Metodologias ativas de ensino para Engenharia.

Nome: Cláudio Dornelles Mello Júnior

E-mail: mellocd@yahoo.com.br

Curriculum Lattes: http://lattes.cnpq.br/5464430592929387

Doutorando em Modelagem Computacional na Universidade Federal do Rio Grande (FURG); mestrado e graduação em Engenharia Elétrica pela Universidade Federal de Santa Maria. Atualmente é Professor Assistente na Universidade Federal de Rio Grande.

Nome: Rodrigo Zelir Azzolin

E-mail: rodrigoazzolin@gmail.com

Curriculum Lattes: http://lattes.cnpq.br/0985488148768235

Doutorado, mestrado e graduação em Engenharia Elétrica pela Universidade Federal de Santa Maria. Atualmente é Professor Adjunto na Universidade Federal do Rio Grande. Tem experiência na área de Engenharia Elétrica, com ênfase em Eletrônica Industrial, Controle de Máquinas Elétricas e Dispositivos de Potência, atuando principalmente, nos seguintes temas: Modelagem e Identificação de Sistemas, Controle de Máquinas Elétricas e Eficiência Energética de sistemas. 